\title{
Analysis of the two-dimensional polydisperse liquid sprays in a laminar boundary layer flow using the similarity transformation method
}

\author{
Ophir Nave $e^{1,2^{*}}$
}

*Correspondence:

naveof@cs.bgu.ac.il

${ }^{1}$ Department

of Mathematics, Ben-Gurion

University of the Negev, PO

Box 653, Beer-Sheva 84105,

Israel

Full list of author information

is available at the end of the article

\begin{abstract}
In this paper we analyze the model that describes the evaporation process of multi-size (polydisperse) of fuel droplets in a laminar boundary layer flow. The spray is described using a probability density function and is based on the well-known sectional approach. The spray is described in two dimensional boundary layer directions that consists of five equations: the continuity equation, the momentum equation, the energy equation, the equation of state and the spray equation. The governing equations are a system of nonlinear partial differential equations (PDE). In order to convert the PDE to ordinary differential equations, we applied the Lees-Dorodnitsyn similarity transformation and the corresponding similar solution based on the compressible stream function. We then solve the model numerically and compare our results to the sectional approach with an experimental data.
\end{abstract}

Keywords: Partial differential equations, Similarity transformation, Polydisperse fuel spray, Laminar boundary layer

\section{Introduction}

The problem of combustion of polydisperse fuel spray in a laminar boundary layer flow is very important from a practical point of view and has a large number of possible applications. The most common description of this problem is discussed by [1-5] when the spray is modeled by the "sectional approach". This method is based on dividing the droplet size domain into sections and dealing only with one integral quantity in each section i.e., number, surface area of droplets, or volume. The advantage of this method is that the integral quantity is conserved within the computational domain and the number of conservation equations is reduced to be simply equal to the number of sections. The natural generalization of this method is to describe the size of the droplets with a probability density function as occurred in this paper [6-10]

Most problems in engineering applications are described by a system of partial differential equations (PDE) which is usually difficult to solve. Using the similarity transformation method $[11,12]$ the system can be converted to an ordinary differential equations (ODE) set by combining two independent variables into a single independent

(c) 2015 Nave. This article is distributed under the terms of the Creative Commons Attribution 4.0 International License (http:// creativecommons.org/licenses/by/4.0/), which permits unrestricted use, distribution, and reproduction in any medium, provided you give appropriate credit to the original author(s) and the source, provide a link to the Creative Commons license, and indicate if changes were made. 
variable [13]. The new set can be solved by a variety of numerical methods. However, with further assumptions that relate to the transport properties, these set of ODEs can be uncoupled mathematically or can have simpler forms, almost similar to the form that is obtained from the incompressible boundary layer analysis. Hence, the simplified ODE set makes it possible to obtain the solution from the already existing solutions of the incompressible analysis and also reduces the computing time of the numerical simulation [14].

In this paper, we apply the Lees-Dorodnitsyn similarity transformation and the corresponding similar solution based on the compressible stream function to the new continuous model. We solve the model numerically and compare our results to the sectional approach model.

\section{Physical assumptions and governing equations}

The physical model is a system of nonlinear partial differential equations in two dimensional $x$ and $y$, steady state, compressible, laminar boundary layers and consists of a multi size (polydipserse) spray of evaporating droplets. The analysis of the spray is restricted to the no slip condition between the droplets and the boundary layer flow field [1].

Generally, in the discrete case, the size distribution of the spray droplets is described by the concentration of discrete droplets of various sizes per unit volume of fluid, $n_{i}(x, y, z, t)$, as a function of the radius $r$, the spatial coordinates $x, y, z$ and of time $t$, where $i=1,2,3, \ldots$ i.e., it is assumed that each droplet consists of an integer multiple of monomers [2]. The concentration conservation equation for each $i$-mer is given by:

$$
\frac{\partial n_{i}}{\partial t}+v \cdot \nabla n_{i}=D_{i} \nabla^{2} n_{i}-E_{i} n_{i}+E_{i+1} n_{i+1}, \quad i=1,2,3, \ldots
$$

By dividing the entire droplet size domain into $m$ arbitrary sections, Tambour [1] defined the integral quantity, $Q_{j}(x, y, z, t)$, to be an integral quantity within the $j$ th section. This is:

$$
Q_{j}(x, y, z, t)=\sum_{i=k_{j-i}+1}^{k_{j}} i^{\gamma} n_{i}(x, y, z, t), \quad j=1,2,3, \ldots, m,
$$

where $k_{j-i}+1$ and $k_{j}$ denote the number monomers in the smallest and largest droplets respectively, in the $j$ th section.

In the continuous case we should replace the summation by integral as follows:

$$
Q(v, t)=\int v^{\gamma} n(v, t) d v
$$

where $v$ is the volume of the droplets.

In our analysis we describe the spray equation in terms of the droplet radius. Hence, in two dimensional $x$ and $y$, the droplets radius are described by the probability density function $n(x, y, r)$, such that the integral over all droplets provides the total number of droplets per unit volume. This quantity is denoted by $Q_{0}$. In general the distribution function of the $i$ th moment following the notation of [15] is:

$$
Q_{\alpha}(x, y)=\int_{0}^{\infty} r^{\alpha} n(x, y, r) d r, \quad \alpha=0,1,2,3 .
$$


For any representation of a droplet size distribution function the assumption is made that the droplets are approximately spherical. Using this assumption, one can see that the moments $Q_{2}$ and $Q_{3}$ represent physical quantities, the knowledge of which is useful for modeling sprays. The surface area of the droplets per unit total volume is $4 \pi Q_{2}$, and the liquid volume is $\frac{4 \pi}{3} Q_{3}$. More details about these four moments can be found in [16].

Under the above assumptions, the governing equations are:

Continuity equation

$$
\frac{\partial(\rho u)}{\partial x}+\frac{\partial(\rho v)}{\partial y}=0
$$

Momentum equations

$$
\begin{aligned}
& \rho u \frac{\partial u}{\partial x}+\rho v \frac{\partial u}{\partial y}=-\frac{\partial p}{\partial x}+\frac{\partial}{\partial y}\left(\mu \frac{\partial u}{\partial y}\right), \\
& \frac{\partial p}{\partial y}=0,
\end{aligned}
$$

Energy equation

$$
\rho u \frac{\partial h}{\partial x}+\rho v \frac{\partial h}{\partial y}=u \frac{\partial p}{\partial x}+\frac{1}{\operatorname{Pr}} \frac{\partial}{\partial y}\left(\mu \frac{\partial h}{\partial y}\right)+v\left(\frac{\partial u}{\partial y}\right)^{2},
$$

Spray equation

$$
\rho u \frac{\partial Q_{\alpha}}{\partial x}+\rho v \frac{\partial Q_{\alpha}}{\partial y}=\rho D \frac{\partial^{2} Q_{\alpha}}{\partial y^{2}}-B Q_{\alpha},
$$

Boundary conditions The boundary conditions at $y=0$ are given by:

$$
\begin{aligned}
& u(x, 0)=0, \quad v(x, 0)=v(x), \quad h(x, 0)=h(x), \quad \frac{\partial h(x, 0)}{\partial y}=0, \\
& u(x, y \rightarrow \infty) \rightarrow U_{e}(x), \quad h(x, y \rightarrow \infty) \rightarrow h_{e}(x), \quad Q_{\alpha}=Q_{\alpha_{0}}, \quad \frac{\partial Q_{\alpha}}{\partial y}=0
\end{aligned}
$$

\section{Nondimensional model and similarity transformation}

In order to convert the model to a non-dimensional one, we define the following nondimensional coordinates as follows:

$$
\begin{aligned}
u^{*} & =\frac{u}{U_{e}}, \quad v^{*}=\frac{v}{U_{e}}, \quad y^{*}=\frac{y}{L}, \quad x^{*}=\frac{x}{L}, \quad h^{*}=\frac{h}{h_{e}}, \\
\mu^{*} & =\frac{\mu}{\mu_{e}}, \quad p^{*}=\frac{p}{\rho U_{e}^{2}}, \quad \rho^{*}=\frac{\rho}{\rho_{e}},
\end{aligned}
$$

The similarity solutions are valid for Reynolds numbers which are large enough, so that the classical boundary-layer assumptions can be applied. Because Reynolds number is a function of $L, R e=\rho_{e} U_{e} L / \mu_{e}$, hence the characteristic value of $L$ can be taken as $10 \mathrm{~cm}$. 
Then, the original Eqs. (2.5)-(2.9) become:

$$
\begin{aligned}
& \frac{\partial\left(\rho^{*} u^{*}\right)}{\partial x^{*}}+\frac{\partial\left(\rho^{*} v^{*}\right)}{\partial y^{*}}=0, \\
& \rho^{*} u^{*} \frac{\partial u^{*}}{\partial x^{*}}+\rho^{*} v^{*} \frac{\partial u^{*}}{\partial y^{*}}=-\frac{\partial p^{*}}{\partial x^{*}}+\frac{1}{\operatorname{Re}} \frac{\partial}{\partial y^{*}}\left(\mu^{*} \frac{\partial u^{*}}{\partial y^{*}}\right), \\
& \frac{\partial p^{*}}{\partial y^{*}}=0, \\
& \rho^{*} u^{*} \frac{\partial h^{*}}{\partial x^{*}}+\rho^{*} v^{*} \frac{\partial h^{*}}{\partial y^{*}}=(\kappa-1) M_{e}^{2} u^{*} \frac{\partial p^{*}}{\partial x^{*}}+\frac{1}{\operatorname{Pr}} \frac{1}{R e} \frac{\partial}{\partial y^{*}}\left(\mu \frac{\partial h^{*}}{\partial y^{*}}\right) \\
& \quad+\bar{v}\left(\frac{\partial u^{*}}{\partial y}\right)^{2}+\frac{(\kappa-1) M_{e}^{2}}{\operatorname{Re}} \bar{v}\left(\frac{\partial u^{*}}{\partial x^{*}}\right), \\
& u^{*} \frac{\partial Q_{\alpha}}{\partial x^{*}}+v^{*} \frac{\partial Q_{\alpha}}{\partial y^{*}}=\frac{D}{U_{e} L} \frac{\partial^{2} Q_{\alpha}}{\partial y^{* 2}}-\frac{L B}{U_{e}} Q_{\alpha},
\end{aligned}
$$

where

$$
\bar{v}=\frac{\mu_{e}}{\rho_{e}}, \quad R e=\frac{\rho_{e} U_{e} L}{\mu_{e}}, \quad \kappa=\frac{C_{p}}{C_{\nu}}, \quad(\kappa-1) M_{e}^{2}=\frac{U_{e}^{2}}{h_{e}} .
$$

\section{The model in terms of stream function}

Our aim in this section is to define the Eqs. (3.2)-(3.6) in terms of stream function. For this purpose, the energy equation can be rewritten in terms of the total enthalpy: $H=h+u^{2} / 2$. For the second step for the similarity transformation and the corresponding similar solutions, the compressible stream function can be defined according to [12] by (Henceforth, asterisk will be omitted from all non-dimensional quantities):

$$
\frac{\partial \Psi}{\partial y}=\rho u, \quad \frac{\partial \Psi}{\partial x}=-\rho v .
$$

The stream function satisfies the continuity Eq. (2.5). First we transform the energy and momentum equations and then we will elaborate on the spray equation.

Substituting Eq. (3.8) into the momentum Eq. (3.3) and into the energy Eq. (3.5) yields these equations in terms of the stream function in the form of:

$$
\begin{gathered}
\frac{\partial \Psi}{\partial y} \frac{\partial}{\partial x}\left(\frac{1}{\rho} \frac{\partial \Psi}{\partial y}\right)-\frac{\partial \Psi}{\partial x} \frac{\partial}{\partial y}\left(\frac{1}{\rho} \frac{\partial \Psi}{\partial y}\right)=-\frac{\partial p}{\partial x}+\frac{\partial}{\partial y}\left[\mu \frac{\partial}{\partial y}\left(\frac{1}{\rho} \frac{\partial \Psi}{\partial y}\right)\right], \\
\frac{\partial \Psi}{\partial y} \frac{\partial H}{\partial x}-\frac{\partial \Psi}{\partial x} \frac{\partial H}{\partial y} \\
=(1-1 / \operatorname{Pr})\left\{\mu\left[\frac{\partial}{\partial y}\left(\frac{1}{\rho} \frac{\partial \Psi}{\partial y}\right)\right]^{2}+\frac{1}{\rho} \frac{\partial \Psi}{\partial y} \frac{\partial}{\partial y}\left[\mu \frac{\partial}{\partial y}\left(\frac{1}{\rho} \frac{\partial \Psi}{\partial y}\right)\right]\right\} \\
\quad+\frac{1}{\operatorname{Pr}} \frac{\partial}{\partial y}\left(\mu \frac{\partial H}{\partial y}\right)^{2} .
\end{gathered}
$$


According to the incompressible boundary layer equations, the dependent variable transformations are introduced as follows:

$$
\begin{aligned}
\Psi(x, y) & =\psi(x) f(\xi, \eta), \\
u(x, y) & =U_{e}(x) \frac{\partial f(\xi, \eta)}{\partial \eta}, \\
Q(x, y) & =Q(\eta) Q_{w}(\xi), \\
H(x, y) & =H_{e}(x) g(\xi, \eta),
\end{aligned}
$$

where the subscript $\eta$ indicates partial differentiation. The form of the enthalpy transformation, Eq. (3.11), states that the compressible boundary layer is expected to be similar with respect to a non-dimensional total enthalpy profile rather than the static enthalpy or temperature profile, as in the case for the incompressible constant property boundary layer.

Let us define the independent variable transformations as follows:

$$
\xi=\xi(x), \quad \eta=\eta(x, y) .
$$

From Eq. (3.8):

$$
\begin{aligned}
\frac{\partial \Psi}{\partial y} & =\psi(x) \frac{\partial \eta}{\partial y} \frac{\partial f(\xi, \eta)}{\partial \eta}=\rho u=\rho U_{e}(x) \frac{\partial f(\xi, \eta)}{\partial \eta}, \\
\Rightarrow & \frac{\partial \eta}{\partial y}=\frac{U_{x} \rho}{\psi(x)} .
\end{aligned}
$$

Integrating Eq. (3.14) yields the expression for $\eta$ as follows:

$$
\eta=\frac{U_{e}(x)}{\psi(x)} \int_{0}^{y} \rho d y
$$

where $\psi(x)$ will be determined from the transformed momentum and energy equations.

Using the conditions at the edge of the boundary layer as reference condition results in

$$
\frac{\mu}{\mu_{e}}=\tilde{C}(\eta) \frac{T}{T_{e}}, \quad(w=1),
$$

which, from $\partial p / \partial y=0$ and the equation of state $\rho \mu=\tilde{C}(\eta) \rho_{e} \mu_{e}$. The coefficient $\tilde{C}(\eta)$ can be assumed as a constant according to the Sutherland viscosity law.

Substituting Eqs. (3.12), (3.15), (3.16) and the equation of state into the momentum Eq. (3.3) and energy Eq. (3.5) yield

$$
\begin{aligned}
& \frac{U_{e}^{2}}{\psi^{2}} \rho_{e} \mu_{e}\left(\tilde{C} f_{\eta \eta}\right)_{\eta}+\frac{\psi_{x}}{\psi} U_{e} f_{\eta \eta}-U_{e_{x}}\left(f_{\eta}\right)^{2}-\frac{1}{\rho_{e} U_{e}} \frac{d p}{d x}=U_{e} \xi_{x}\left(f_{\eta} f_{\xi \eta}-f_{\eta \eta} f_{\xi}\right) \\
& \frac{U_{e}^{2}}{\psi^{2}} H_{e} \rho_{e} \mu_{e}\left(\tilde{C} g_{\eta}\right)_{\eta}+\frac{\psi_{x}}{\psi} H_{e} \operatorname{Prf} g_{\eta}-P r H_{e_{x}} f_{\eta} g+(\operatorname{Pr}-1) \frac{U_{e}^{3}}{\psi^{2}} \rho_{e} \mu_{e}\left(\tilde{C} f_{\eta} f_{\eta \eta}\right)_{\eta} \\
& \quad=H_{e} \xi_{x}\left(f_{\eta} g_{\xi}-f_{\xi} g_{\eta}\right) .
\end{aligned}
$$


We assumed that $\tilde{C}$ is constant. The flow is assumed to be similar, i.e., $f=f(\eta)$, $g=g(\eta)=H / H_{e}\left(H_{e}\right.$ is constant) such that the RHS of the momentum and energy equation becomes zero. In addition, we assumed that the total enthalpy at the boundary layer edge is constant, i.e., $H_{e_{x}}=0$. Since $H_{e}=h_{e}+0.5 U_{e}^{2}$, both the static enthalpy and the velocity can vary across the edge of the boundary layer. In the momentum equation, we replace the pressure gradient term by using Euler's equation at the edge of the boundary layer, i.e.,

$$
U_{x}=-\frac{1}{\rho_{e} U_{e}} \frac{d p}{d x}
$$

Using Eq. (3.19) for the momentum equation, together with the stagnation enthalpy, the momentum and energy equations become respectively:

$$
\begin{aligned}
& f^{\prime \prime \prime}+\frac{\psi \psi_{x}}{\tilde{C} \rho_{e} \mu_{e} U_{e}} \not f^{\prime}+\frac{\psi^{2} U_{e_{x}}}{\tilde{C} \rho_{e} \mu_{e} U_{e}^{2}}\left(\frac{\rho_{e}}{\rho}-\left(f^{\prime}\right)^{2}\right)=0, \\
& g^{\prime \prime}+\frac{\psi \psi_{x}}{\tilde{C} \rho_{e} \mu_{e} U_{e}} \operatorname{Prg}^{\prime}=(1-\operatorname{Pr}) \frac{U_{e}^{2}}{H_{e}}\left(f^{\prime} f^{\prime \prime}\right)^{\prime},
\end{aligned}
$$

where, the prime denotes ordinary differentiation with respect to $\eta$. The similarity conditions from Eqs. (3.20)-(3.21) are

$$
\begin{aligned}
& \frac{\psi \psi_{x}}{\tilde{C} \rho_{e} \mu_{e} U_{e}}=1, \\
& \frac{\psi^{2} U_{e_{x}}}{\tilde{C} \rho_{e} \mu_{e} U_{e}^{2}}\left(\frac{\rho_{e}}{\rho}-\left(f^{\prime}\right)^{2}\right)=\text { function of } \eta \text { only, } \\
& \frac{U_{e}^{2}}{H_{e}}=1 .
\end{aligned}
$$

Integrating Eq. (3.22) results in:

$$
\psi(x)=\sqrt{2 \int_{0}^{x} \rho_{e} \mu_{e} U_{e} d x} \equiv \sqrt{2 \xi(x)} .
$$

Substituting Eq. (3.25) into (3.15) results in:

$$
\eta=\frac{U_{e}}{\sqrt{2 \int_{0}^{x} \tilde{C} \rho_{e} \mu_{e} U_{e} d x}} \int_{0}^{y} \rho d y .
$$

Since $\xi=\xi(x)$ and

$$
\xi=\int_{0}^{x} \tilde{C} \rho_{e} \mu_{e} U_{e} d x
$$


then Eq. (3.26) becomes

$$
\eta=\frac{U_{e}}{\sqrt{2 \xi}} \int_{0}^{y} \rho d y
$$

The transformation given in Eqs. (3.27)-(3.28) is called the Illingworth-levy transformation.

For the case of $H_{e}=$ const, using the definition of $g(\eta)$, and since $u / U_{e}=f^{\prime}$ and with the added assumption that the pressure is constant across the boundary layer, the final form of the momentum Eq. (3.20) and energy Eq. (3.21) are:

$$
\begin{aligned}
& f^{\prime \prime \prime}+\not f^{\prime \prime}+\frac{2 \xi}{U_{e}} \frac{d U_{e}}{d \xi}\left(1+\frac{(\kappa-1) M_{e}^{2}}{2}\right)\left(g-\left(f^{\prime}\right)^{2}\right)=0, \\
& g^{\prime \prime}+\operatorname{Prfg} g^{\prime}=\frac{2(\kappa-1) M_{e}^{2}}{2+(\kappa-1) M_{e}^{2}}(1-\operatorname{Pr})\left(f^{\prime} f^{\prime \prime}\right)^{\prime} .
\end{aligned}
$$

The next step is to transform the spray Eq. (3.6) under the above assumptions, using the stream function, and the Illingworth-levy transformation. For this purpose it is necessary to multiply the spray equation by the density $\rho$.

$$
\frac{\partial \Psi}{\partial y} \frac{\partial Q_{\alpha}}{\partial x}-\frac{\partial \Psi}{\partial x} \frac{\partial Q_{\alpha}}{\partial y}=\frac{\rho D}{U_{e} L} \frac{\partial^{2} Q_{\alpha}}{\partial y^{2}}-\frac{\rho L B}{U_{e}} Q_{\alpha} .
$$

Using the transformation (3.27)-(3.28) we obtain

$$
\frac{\partial \Psi}{\partial \eta} \frac{\partial Q_{\alpha}}{\partial \xi}-\frac{\partial \Psi}{\partial \xi} \frac{\partial Q_{\alpha}}{\partial \eta}=\frac{\rho^{2} D}{\tilde{C} \mu_{e} \rho_{e} U_{e} L \sqrt{s \xi}} \frac{\partial^{2} Q_{\alpha}}{\partial \eta^{2}}-\frac{L B \sqrt{2 \xi}}{\tilde{C} U_{e}^{3} \mu_{e} \rho_{e}} Q_{\alpha} .
$$

Next, using the transformation (3.27)-(3.28) we obtain

$$
\Psi=\sqrt{2 s \xi} f .
$$

Substituting Eq. (3.33) into (3.32) and using the relation $Q_{\alpha}(\eta)=Q_{\alpha} / Q_{\alpha, w}(s)$, according to [1], the spray Eq. (3.32) transforms to:

$$
\Lambda Q_{\alpha}^{\prime \prime}+\Delta f Q_{\alpha}^{\prime}=\left(\Omega f^{\prime}+\Gamma\right) Q_{\alpha}
$$

where

$$
\Lambda=\frac{\rho^{2} D}{\tilde{C} \mu_{e} \rho_{e} U_{e} L \sqrt{2 \xi}}, \quad \Omega=\frac{d Q_{\alpha, w}}{d \xi} \sqrt{2 \xi}, \quad \Gamma=\frac{L B \sqrt{2 \xi}}{\tilde{C} U_{e}^{3} \mu_{e} \rho_{e}}, \quad \Delta=\frac{1}{\sqrt{2 \xi}} .
$$

The governing equations of the model that describe the vaporization of polydisperse fuel spray in a laminar boundary layer flow, in terms of probability density function, are Eqs. (3.29)-(3.30) and (3.34), with the boundary conditions at the surface for similar solutions to be exist are:

$$
\text { at } \quad \eta=0: f=0, \quad f^{\prime}=0, \quad Q_{\alpha, w}=1 .
$$


The boundary conditions related to the energy equation are

$$
\text { at } \quad \eta=0: g=\text { const }=g_{w}, \text { or } \quad g^{\prime}=0 .
$$

The outer boundary conditions are

$$
\text { at } \quad \eta \rightarrow \infty: f^{\prime} \rightarrow 1, \quad g \rightarrow 1, \quad Q_{\alpha, w} \rightarrow 0 .
$$

That is, the wall temperature is assumed to be known, and the initial size distribution of the droplets produced by the atomizer is known.

\section{Discussion}

The purpose of the atomization is to disperse the droplets into the oxidizer and to increase the surface area of the droplets. This process of atomization enhanced the heat and mass transfer during the combustion processes.

In our analysis we compared among three different initial size distributions of droplets: Non symmetrical, Symmetrical and Uniform distribution. Each distribution refers to the surface area of the droplets. In order to compare our continuous model to the sectional approximation model, we have taken into account five sections, as can be seen in Table 1 .

Figures 1, 2 present the downstream changes in the surface area distribution of droplets for continuous and sectional approximation models, as compared with the experimental results, correspondingly. At the beginning of the vaporization process the sectional approach more closely modeled the experimental results (for small droplets). For large droplets the continuous approach more closely modeled the experimental results. Our results show a substantial decrease in the surface are of the droplets both in the higher section and in the lower section.

According to Figs. 3, 4 for the non symmetrical initial distribution, as the free stream temperature increases, the droplets boundary layer thickness is decreased both for the sectional approximation and for the continuous model correspondingly. The same conclusion can be drawn for the symmetrical initial distribution and for the uniform initial distribution, see Figs. 5, 6 and 7, 8 correspondingly. From these figures one can see that the surface area of the droplets in section $V$ is decreased significantly compared to section $I$.

\section{Comparison with experimental results}

In our analysis we compared our results with experimental data given in Ref. [17]. In the experimental data the liquid phase (the fuel) sprayed in a high pressure environment decomposed and then form a polydisperse fuel spray of droplets. In our notations

\begin{tabular}{|c|c|c|c|c|}
\hline Section number & Droplet diameter $(\mu \mathrm{m})$ & Uniform & Symmetrical & Nonsymmetrical \\
\hline I & $<1$ & 0.2 & 0.5 & 0.48 \\
\hline$\|$ & $1-3$ & 0.2 & 0.8 & 0.66 \\
\hline III & $3-5$ & 0.2 & 0.6 & 0.32 \\
\hline IV & $5-6$ & 0.2 & 0.8 & 0.56 \\
\hline V & $6-9$ & 0.2 & 0.5 & 0.63 \\
\hline
\end{tabular}

Table 1 Initial surface area distribution 

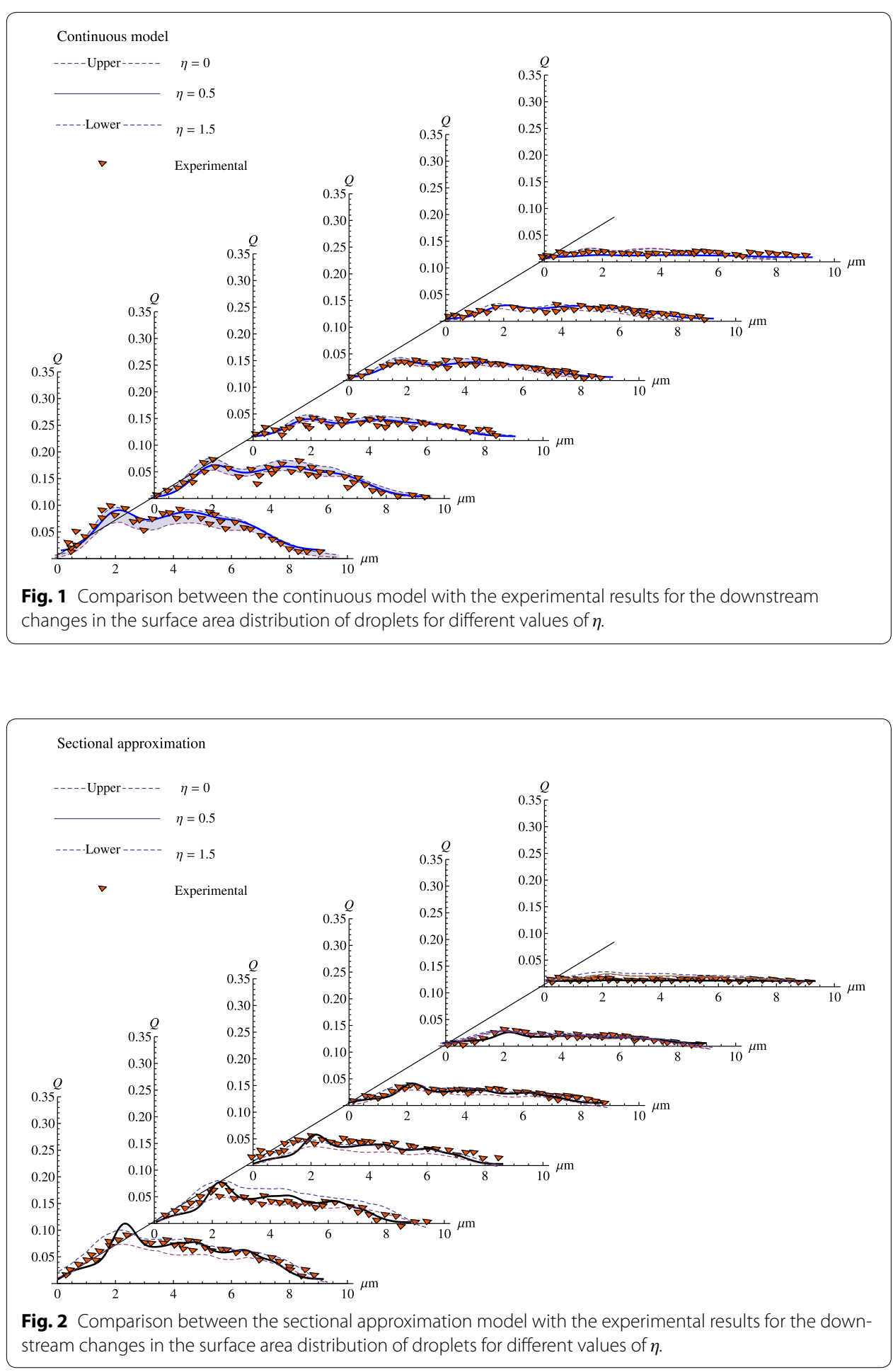

the distribution function for the droplets size is $n(2 r, t)$ and in dimensionless form is as follows:

$$
n(2 r, \tau)=\frac{81 \pi^{2}}{128} r\left[\tau\left(1-\zeta \tau^{2}\right)+r^{2}\right] e^{\frac{9 \pi}{16 r_{0}^{2}}\left[\tau\left(1-\zeta \tau^{2}\right)+r^{2}\right]}
$$




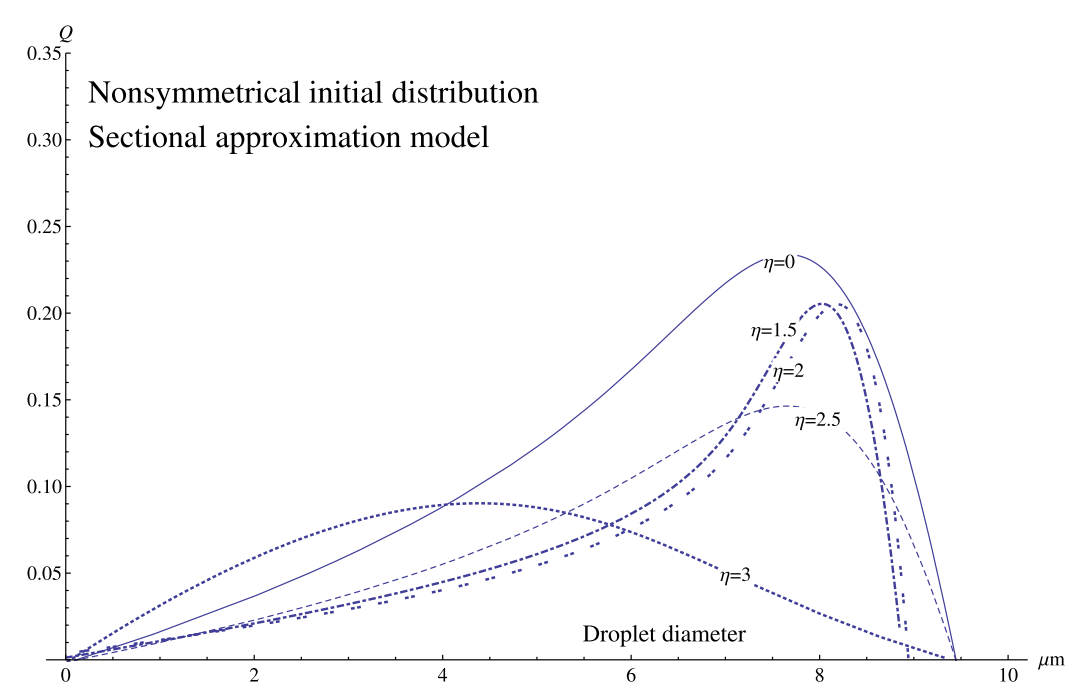

Fig. 3 Surface area distribution of droplets as a function of the distance from the wall, for an axial position $\bar{s}=1$ for the sectional approximation model for different values of $\eta$.

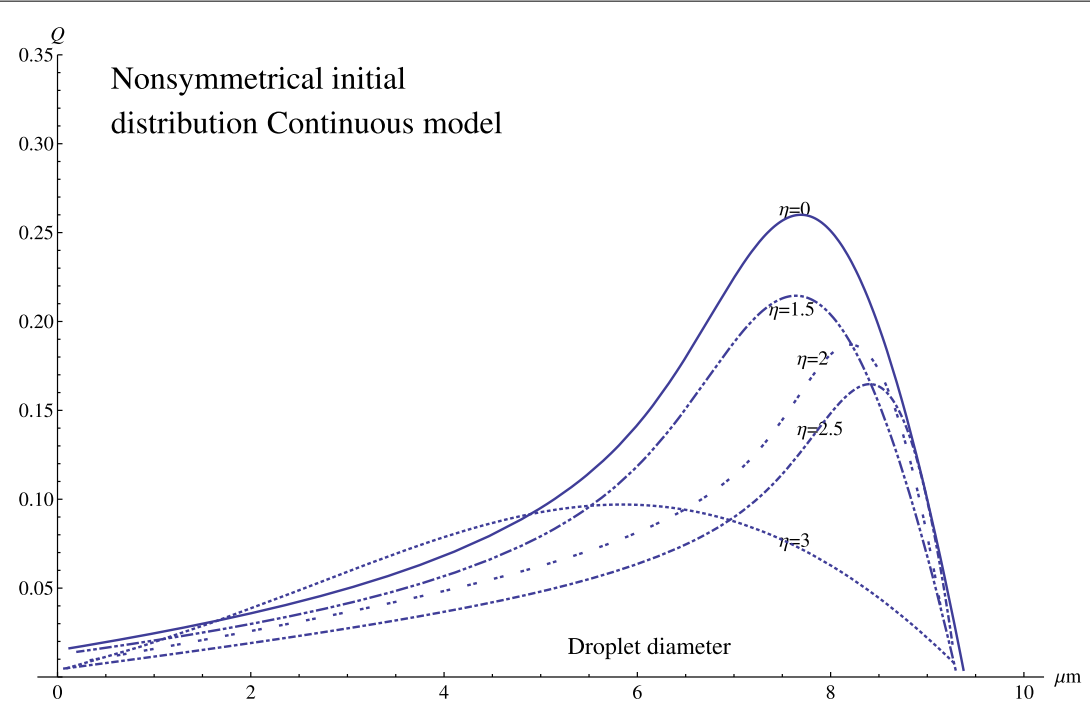

Fig. 4 Surface area distribution of droplets as a function of the distance from the wall, for an axial position $\bar{s}=1$ for the continuous model for different values of $\eta$.

where $\tau$ is the dimensionless time, $r_{0}$ dimensionless initial radius and $\zeta$ constant inefficiency parameter (for more details refer to [17]). As presented in Figs. 1, 2 the evaporation rate decreases gradually rather than abruptly (the decreases of the droplet moment Q) (this results agree with the observation in [17]). In order to compare quantitatively our results with experimental results we should re-normalized our initial droplet radius to the initial droplet radius as presented in [17]. In Fig. 9 we presented the relative error of our results compared to experimental data for the same initial dimensionless droplet radius. The expression for the relative error given by $\left(n_{\text {theoretical }}-n_{\text {experimental }}\right) / n_{\text {experimental }}$. For example, at time $=0.6$ the relative error between the continuous model (non-symmetrical PDF) and the sectional approach model is $5 \%$. 


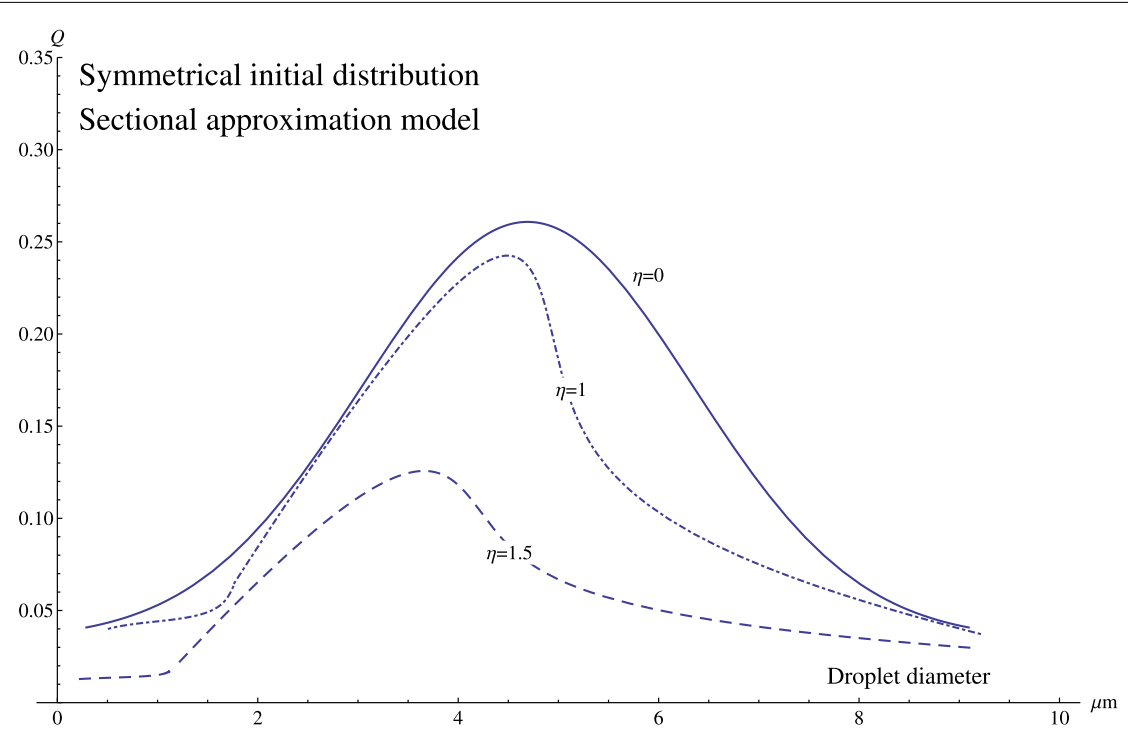

Fig. 5 Surface area distribution of droplets as a functionggg of the distance from the wall, for an axial position $\bar{s}=1$ for the sectional approximation model for different values of $\eta$.

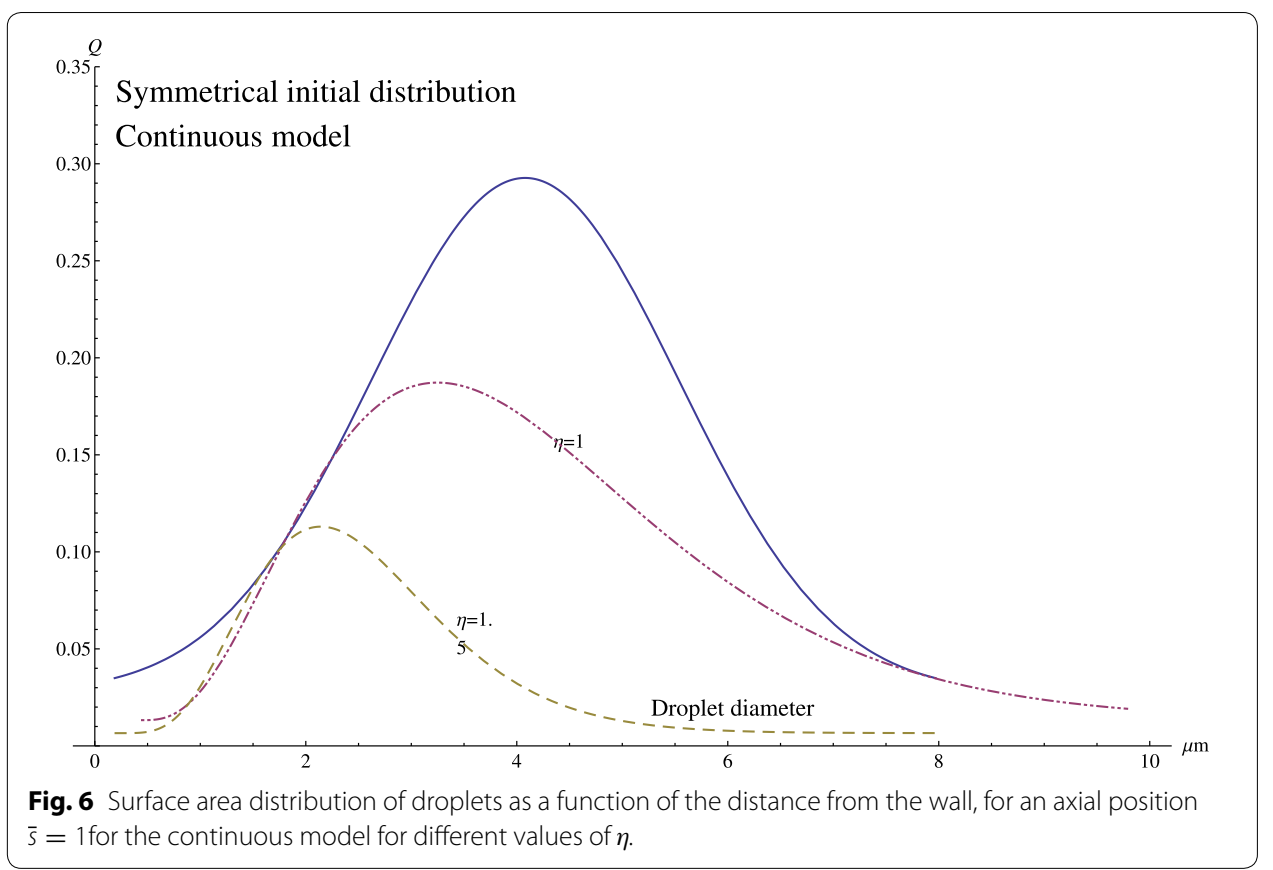

\section{Conclusion}

In this research paper we developed a model that describe the evaporation process of multi-size (polydisperse) of fuel droplets in a laminar boundary layer flow. We rewrite the discrete model that describe this process and based on the well-known the sectional approach model. In order to analyze the model we transfer the discrete model to a continuous one using a probability density function, i.e., the size distribution of the spray droplets, which is a polydisperse spray droplets, described by a four moments model 


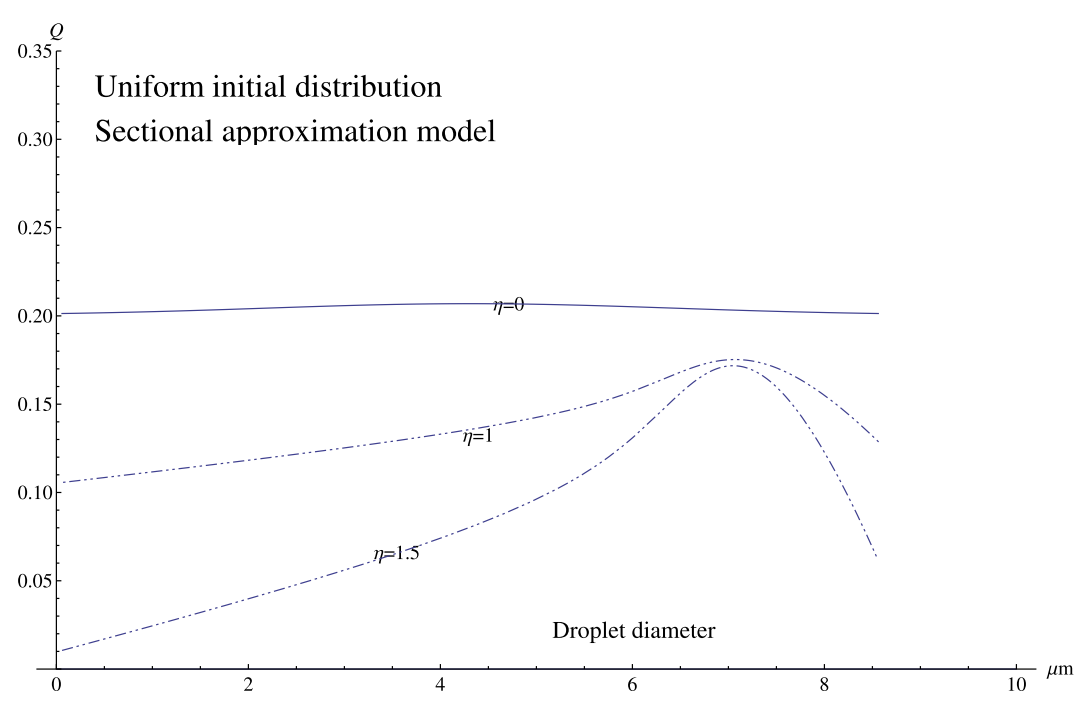

Fig. 7 Surface area distribution of droplets as a function of the distance from the wall, for an axial position $\bar{s}=1$ for the sectional approximation model for different values of $\eta$.

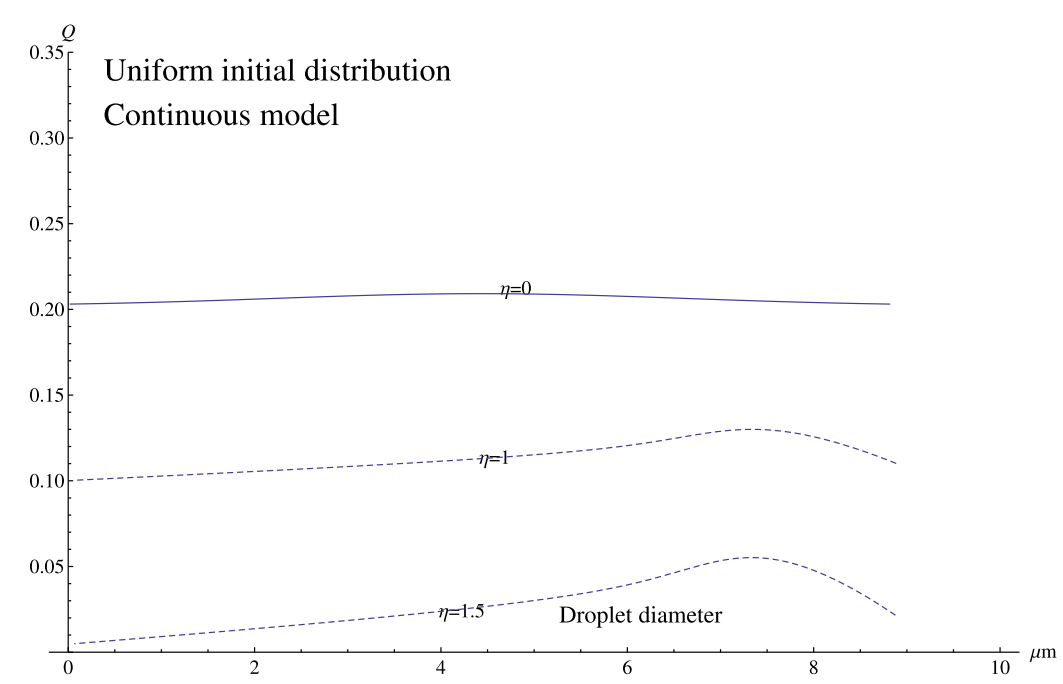

Fig. 8 Surface area distribution of droplets as a function of the distance from the wall, for an axial position $\bar{s}=1$ for the continuous model for different values of $\eta$.

using the quantity $Q_{i}$. The analysis of the spray restricted to a no slip condition between the droplets and the boundary layer flow filed. The physical model includes a system of five partial differential equations. We applied the Lees-Dorodnitsyn similarity transformation and the system of the governing equations transform to an ordinary differential equation. This transformation enable one to apply an asymptotic methods and even an analytical methods such as the singular perturbed homotopy analysis method (SPHAM). Our future work is to apply the SPHAM to the continuous model and obtain an analytical solution for the physical dynamical variables of the system.

We compared the theoretical results to the experimental data (in contrast to [1]), as can be seen in Table 2. Additionally, we have rewritten the model that was presented 


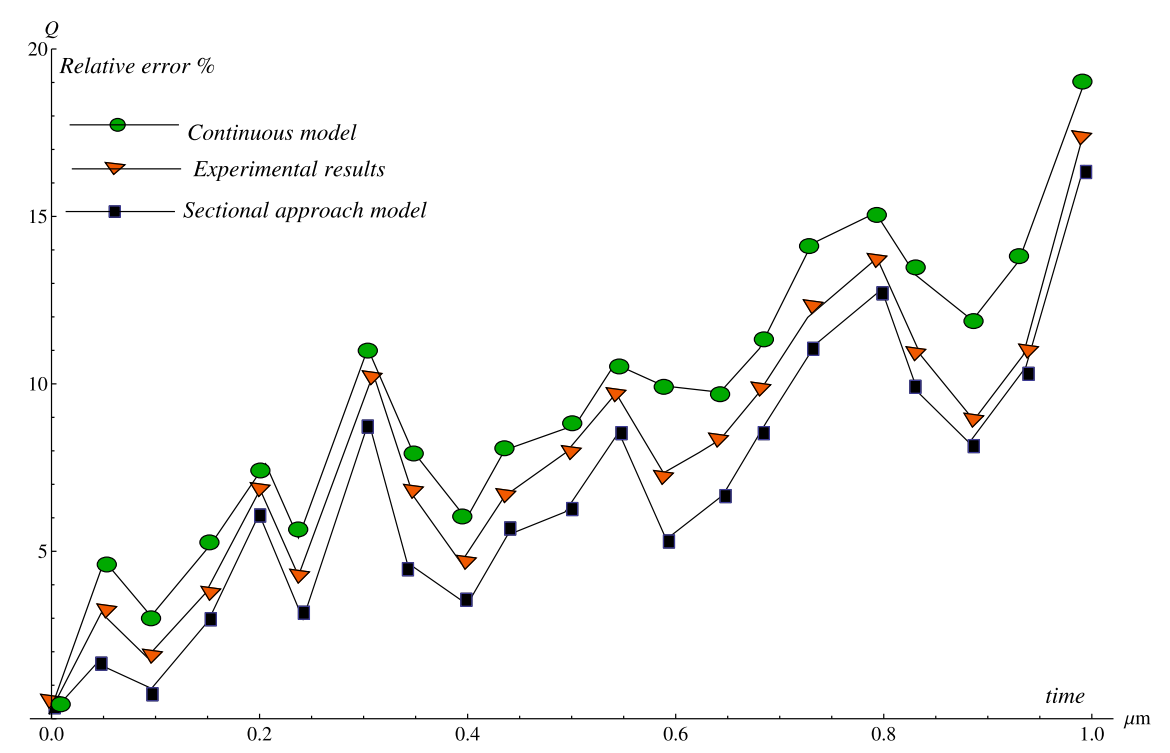

Fig. 9 Relative error. Qualitative comparison between the continuous model using non-symmetrical PDF and experimental data in percent.

Table 2 Section number and sectional coefficients normalized by $E(T)$, the surface recession rate of a $65 \mu \mathrm{m}$ droplets

\begin{tabular}{llll}
\hline Section number & Droplet diameter $(\mu \mathrm{m})$ & $C_{j} / E(T)$ & $\mathbf{B}_{j, j+1} / E(T)$ \\
\hline I & $<1$ & 0.454 & 0.0692 \\
II & $1-3$ & 0.987 & 0.0018 \\
III & $3-5$ & 0.657 & 0.0875 \\
IV & $5-6$ & 0.342 & 0.0787 \\
V & $6-9$ & 0.566 & - \\
\hline
\end{tabular}

in [1] in a continuous form. This reformulation not only reduced the complexity of the model but also reduced the computation time, which is very important from a practical point of view. This change is particularly evident in the spray equation, which in our model is merely one equation!. In conclusion, we demonstrate in this paper how the surface area of the droplets in polydisperse fuel spray has been changed as a function of the temperature and the distance from the wall.

\section{Nomenclature}

$B \quad$ evaporation coefficient

C specific heat capacity

$D \quad$ diffusion coefficient of droplets

$E_{i} \quad$ frequency of the molecule evaporation from an $i$-mer droplet

$h \quad$ enthalpy

$L \quad$ characteristic longitudinal direction

$M \quad$ Mach number

$n \quad$ number of droplets per unit volume

$p \quad$ pressure 


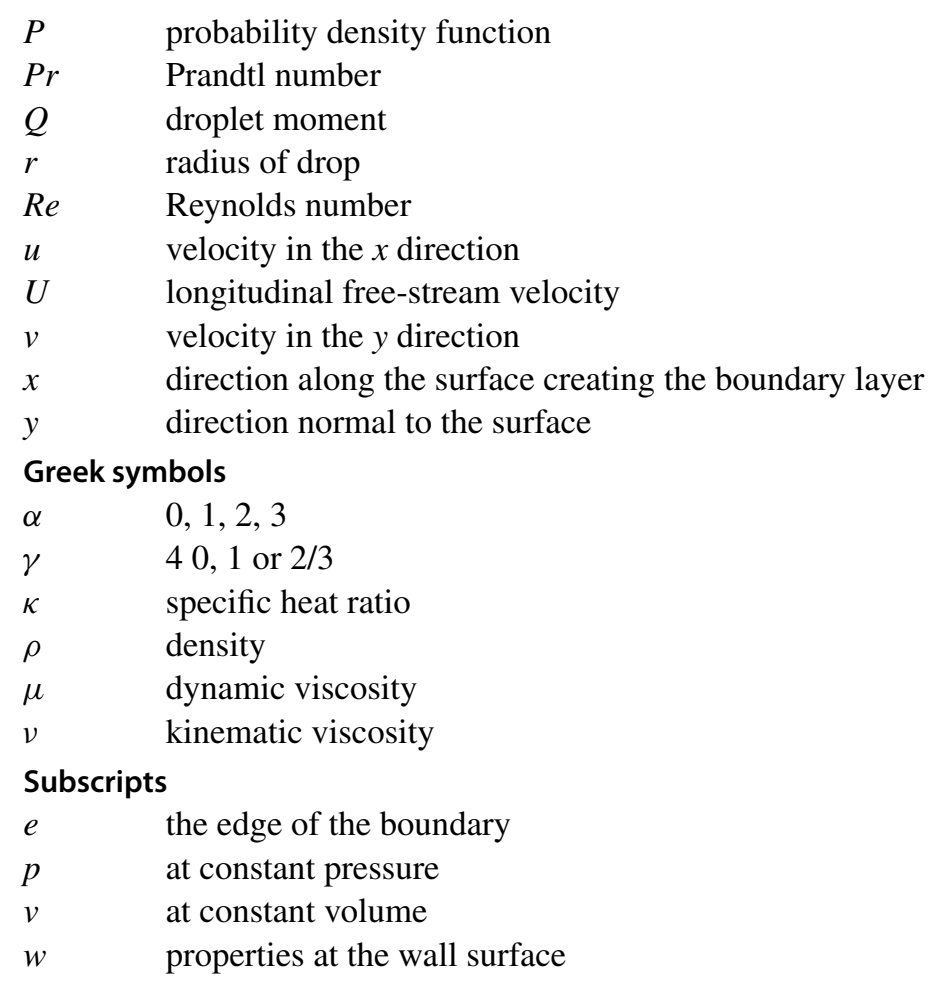

Compliance with ethical guidelines

Competing interests

The authors declare that they have no competing interest.

\section{Author details}

${ }^{1}$ Department of Mathematics, Ben-Gurion University of the Negev, PO Box 653, Beer-Sheva 84105, Israel. ${ }^{2}$ Jerusalem College of Technology (JCT), Jerusalem, Israel.

Received: 18 May 2015 Accepted: 30 July 2015

Published online: 12 August 2015

\section{References}

1. Tambour Y (1984) Vaporization of polydisperse fuel sprays in a laminar boundary layer flow: a sectional approach. Comb Flame 58:103-114

2. Tambour Y (1985) A Lagrangian sectional approach for simulating droplet size distribution vaporizing fuel sprays in a turbulent jet. Comb Flame 60:15-28

3. Tambour Y, Zehavi S (1993) Derivation of near-field sectional equations for the dynamics of polydisperse spray flows: an analysis of the relaxation zone behind a normal shock wave. Comb Flame 95:383-409

4. Katoshevski D, Tambour Y (1993) A theoretical study of polydisperse liquid-sprays in a free shear-layer flow. Phys Fluid A 5:3085-3098

5. Greenberg JB (1989) The Burke-Schumann diffusion flame-with fuel spray injection. Comb Flame 77:229-240

6. Sirignano WA (2010) Fluid dynamics and transport of droplets and sprays, Cambridge University Press, England

7. Inamura T, Tamura H, Sakamoto H (2003) Characteristics of liquid film and spray injected from Swirl Coaxial Injector. J Propuls Power 19:632-639

8. Sureshkumar R, Kale SR, Dhar PL (2008) Heat and mass transfer processes between a water spray and ambient air II. Simulations 28:361-371

9. Girarda F, Meillota E, Vincentc S, Caltagironeb JP, Bianchia L (2015) Contributions to heat and mass transfer between a plasma jet and droplets in suspension plasma spraying. Surf Coat Technol 268:278-283

10. Sazhin S (2014) Droplets and sprays. Springer, London

11. Bertin JJ (1994) Hypersonic aerothermodynamics, AIAA Education Series, Washington, DC

12. Anderson JD (2006) Hypersonic and high temperature gas dynamics. American Institute of Aeronautic and Astronautic, AIAA Education Series, Reston, VA

13. Shateyi S, Marewo GT (2013) A new numerical approach for the Laminar boundary layerflow and heat transfer along stretching cylinder embedded in a porous medium with variable Thermal Conductivity. J Appl Math 13:1-7

14. Kalikhman LE, Potapov AV (1976) Generalized variables in boundary-layer theory. Fluid Dyn 11:789-790 
15. Adam S, Schnerr GH (1997) Instabilities and bifurcations of non-equilibrium two-phase flows. J Fluid Mechan 348(1):1-28

16. Sowa WA (1992) Interpreting mean drop diameters using distribution moments. Atomization Sprays 2:1-15

17. Choudhury PR, Gerstein M (1982) In: Nineteeth Symposium International on Combustion. The Combustion Institute, Pittsburg, pp 993-997

18. Polymeropoulos CE (1973) Steady state vaporization and ignition of liquid spheres. Comb Sci Technol 8:111-112

19. Abramzon B, Sirignano WA (1989) Droplet vaporization model for spray combustion calculations. Int J Heat Mass Transf 32:1605-1618

Submit your manuscript to a SpringerOpen ${ }^{\circ}$ journal and benefit from:

- Convenient online submission

- Rigorous peer review

- Immediate publication on acceptance

- Open access: articles freely available online

- High visibility within the field

- Retaining the copyright to your article

Submit your next manuscript at $>$ springeropen.com 